\title{
Tomographie de fluorescence DUV sur des systèmes d'intérêt biologique
}

Matthieu Réfrégiers (matthieu.refregiers@synchrotron-soleil.fr) ${ }^{(1)}$, Frédéric Jamme ${ }^{(1,2)}$,

Slavka Kascakova ${ }^{(1)}$ et Alain Buléon ${ }^{(2)}$

(1) Ligne DISCO, Synchroton SOLEIL, L’Orme des Merisiers, 91192 Gif-sur-Yvette

(2) Cepia, Institut national de la recherche agronomique (INRA), BP 71627, 44316 Nantes

L'utilisation de la lumière synchrotron en ultraviolet lointain (DUV, de longueur d'onde comprise entre 120 et $300 \mathrm{~nm}$ ) pour exciter la fluorescence des molécules en microscopie, permet de s'affranchir de marqueurs de contrastes.

Un microscope inversé DUV est installé à SOLEIL sur la ligne de lumière DISCO. La motorisation axiale de l'échantillon et le traitement du signal par déconvolution rendent possible I'obtention de tomogrammes avec des résolutions de l'ordre de $100 \mathrm{~nm}$.

Deux applications biologiques sont présentées ici : l'activité d'une enzyme, l'amylase, sur un grain d'amidon; et le suivi de l'entrée de nanoparticules dans des cellules cancéreuses.

Les termes suivis d'un astérisque $\left(^{\star}\right)$ sont définis dans le glossaire.

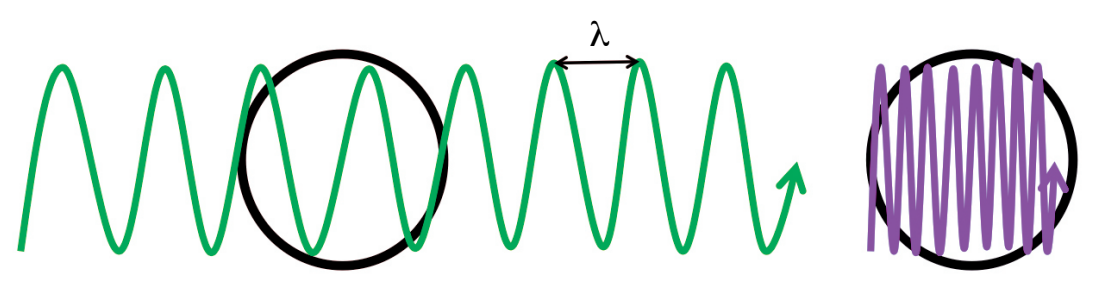

1. Comparaison entre une onde visible (en vert) et une onde ultraviolette (en violet).

Pourquoi les ultraviolets lointains, pourquoi le synchrotron?

La taille des objets que la lumière peut distinguer dépend de la longueur d'onde ( $\lambda$ ) utilisée (fig. 1). D'après les critères de Rayleigh, la distance minimale entre deux points discriminables est directement liée à la longueur d'onde. En microscopie, la loi d'Abbe-Zeiss, qui date du milieu du XIX ${ }^{\mathrm{e}}$ siècle, précise que la distance entre deux objets doit être supérieure à un tiers de la longueur d'onde pour qu'ils soient distinguables. C'est ainsi que Köhler proposa, au début du $\mathrm{XX}^{\mathrm{e}}$ siècle, de réduire la longueur d'onde pour mieux imager les objets de petite taille que l'on trouve dans une cellule de mammifere. Donc, en théorie, plus la longueur d'onde est courte, meilleure est la résolution. C'est ainsi que les rayons $\mathrm{X}$ permettent d'imager des distances atomiques dans des molécules. Les ultraviolets sont un bon compromis entre la lumière visible de grande longueur d'onde et les rayons $\mathrm{X}$ qui peuvent dégrader la matière biologique.

L'utilisation du rayonnement synchrotron comme source d'excitation sur la ligne de lumière DISCO donne accès à un continuum de longueurs d'onde allant du visible à la limite de transmission de l'air (environ $170 \mathrm{~nm}$ ) pour exciter les échantillons, avec une amélioration notable de la résolution. De nombreuses petites molécules d'intérêt biologique ou chimique présentent une forte fluorescence ou luminescence lorsqu'elles sont excitées par des ultraviolets lointains. La fluorescence étant une signature spécifique des molécules, sa détection permet une meilleure sélectivité que l'imagerie par absorption.

\section{Principe de la méthode}

En microscopie de fluorescence plein champ, un rayonnement lumineux est focalisé par des optiques sur un échantillon. La lumière réémise par l'échantillon à des énergies plus basses est collectée vers un détecteur, afin de reconstituer une image d'émission. Les imperfections des optiques utilisées et de la source lumineuse font que l'on acquiert des images floues. De plus, la lumière collectée provient de tous les plans de l'échantillon. Ainsi, on obtient une image intégrée, somme d'images bidimensionnelles ; il devient nécessaire d'utiliser un outil mathématique pour reconstituer un objet en trois dimensions avec une meilleure résolution spatiale : la déconvolution.

Les théories de la déconvolution datent de la Seconde Guerre mondiale ; elles étaient d'abord utilisées dans les domaines 

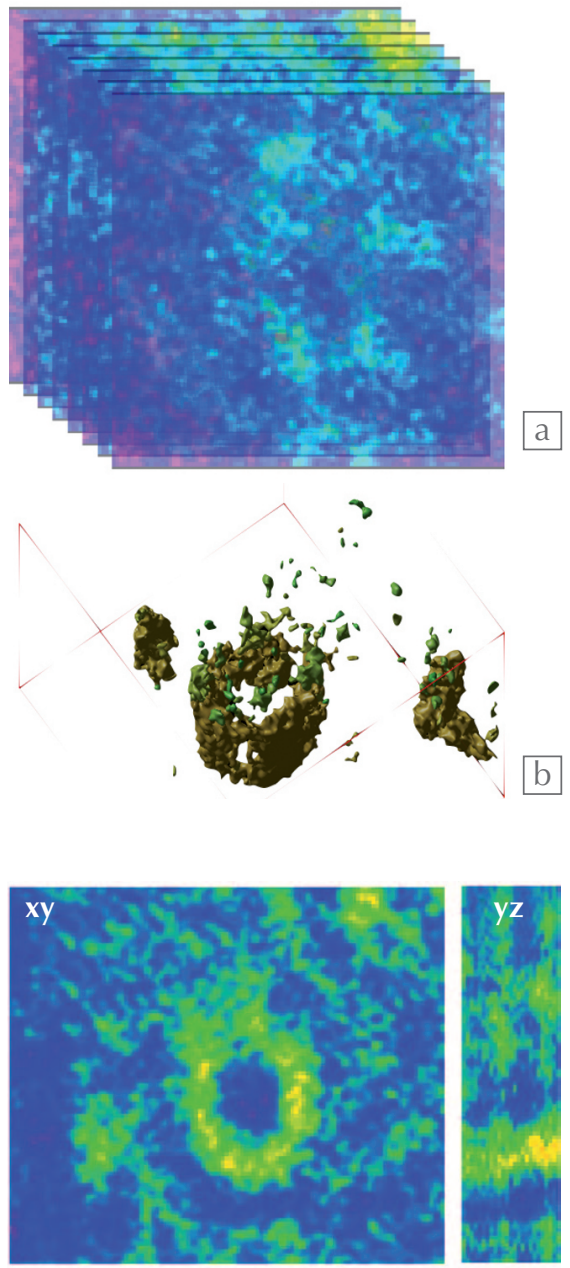

$\mathbf{X Z}$

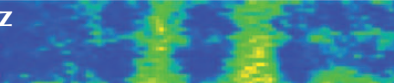

2. Différents modes de représentation bidimensionnelle d'un objet tridimensionnel.

La représentation en plans sériés (a) n'est utile qu'avec une interaction dynamique permettant de passer d'un plan à l'autre. La reconstruction surfacique en trois dimensions (b), bien qu'esthétique, fausse les distances. Seule la représentation en trois projections (c) permet de comprendre la nature tridimensionnelle d'un objet.

\section{Glossaire}

Amylase : enzyme qui dégrade les polysaccharides en disaccharides.

Cellules immortalisées : lignée de cellules possédant un potentiel de multiplication infini, sans être forcément cancérigènes.

PSF : “Point Spread Function” (littéralement fonction d'étalement d'un point par l'appareil), qui décrit la réponse d'un système d'imagerie à une source ponctuelle, incluant l'effet des aberrations.

Tryptophane : acide aminé aromatique, brique élémentaire des protéines. Après excitation dans le DUV, il réémet efficacement une fluorescence ultraviolette.

\section{>>}

de la prédiction météorologique et de l'économie, puis elles ont été appliquées à la sismologie. En traitement du signal physique, les premières approches ont concerné l'électronique. En traitement d'images, les télescopes ont été les premiers bénéficiaires.

La déconvolution améliore la résolution spatiale, le rapport signal sur bruit, et repositionne spatialement l'information afin d'obtenir une image en trois dimensions. La déconvolution en aveugle ou théorique est une technique de restauration d'images issue de l'astronomie, où il n'est pas possible de déterminer une fonction instrumentale et où les objets à étudier sont par nature ponctuels. Ici, au contraire, on peut calculer la fonction instrumentale à partir des paramètres connus de l'instrument (pour un microscope, le grandissement de l'objectif, son milieu d'immersion, son ouverture numérique, le temps d'exposition, les longueurs d'onde d'excitation et d'émission, par exemple). La déconvolution théorique peut être utilisée en microscopie de fluorescence, mais reste gourmande en calculs et, sur des objets non ponctuels, perd en efficacité. C'est pourquoi il est plus rigoureux de mesurer la $\mathrm{PSF}^{*}$ ("Point Spread Function") propre à l'instrument utilisé, comme première étape de déconvolution.

En microscopie de fluorescence, les images sont des plans sériés suivant l'axe Z (fig. 2a). Une image est dégradée par la diffraction et le bruit. La diffraction est due en grande partie à l'émission de fluorescence défocalisée qui se superpose à l'image du plan focal, et le bruit à la fluctuation du flux de photons incidents.
Chaque fluorophore (composé fluorescent) peut être considéré comme une source ponctuelle de lumière ; les images sont donc issues de la somme des contributions des fluorophores, chacune étant représentée par une PSF positionnée à un endroit différent et avec un poids dépendant de l'intensité de fluorescence. La déconvolution permet, à partir d'une image de fluorescence, de repositionner virtuellement dans son plan d'origine la lumière qui s'est propagée : donc de reconstruire une image au plus proche de la réalité. En calculant la fonction de réponse ponctuelle de l'instrument et en appliquant son inverse, on obtient une image restaurée débruitée, dont on peut ensuite reconstruire la structure tridimensionnelle.

Différents modes de présentation d'un tomogramme existent (fig. 2). Le plus simple consiste à présenter des projections suivant les plans XY, XZ et YZ de l'objet tridimensionnel. Des reconstructions avec interpolation des plans manquants sont également possibles, avec rendu surfacique ou volumique. Tous ces modes de représentation sont communs aux imageries tridimensionnelles.

\section{Technique}

Des matrices tridimensionnelles d'images plein champ sont acquises sur un microscope inversé [2] dont l'axe $\mathrm{Z}$ est motorisé (fig. 3). Les nanodéplacements le long de l'axe $Z$ permettent d'enregistrer une série d'images de fluorescence après excitation UV, formant ainsi un cube d'informations. L'ensemble du système est piloté par un logiciel open source: $\mu$ manager [1].

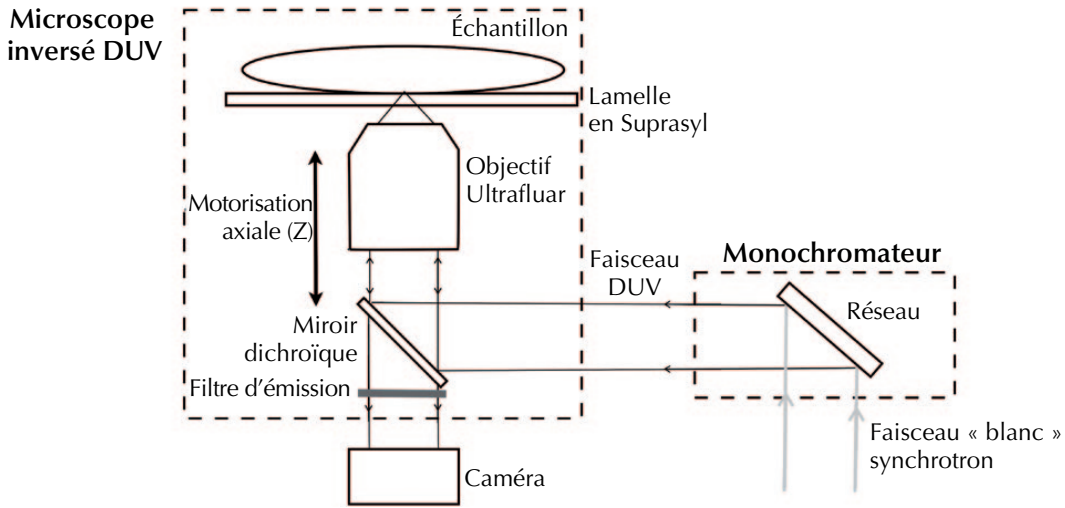

3. Schéma de principe du microscope inversé DUV de la ligne de lumière DISCO du synchrotron SOLEIL. 
La fonction de réponse de l'appareil est enregistrée en prenant comme modèle des billes de polymère fluorescent dans les ultraviolets, de $150 \mathrm{~nm}$ de diamètre. L'analyse et la déconvolution spatiale sont réalisées grâce au logiciel Huygens Deconvolution (SVI, Hilversum, NL).

\section{Premier exemple : activité d'une amylase* sur un support solide}

L'amidon est un glucide de réserve des végétaux. Il constitue une matière première abondante, renouvelable et biodégradable, utilisée à la fois dans l'industrie alimentaire comme source d'énergie, mais aussi dans de nombreuses applications non alimentaires. Actuellement, on observe une diminution des réserves d'énergie fossile et une augmentation des gaz à effet de serre. Les produits issus de l'agriculture, tels que l'amidon, sont donc utilisés pour la production de matériaux plastiques verts et de biocarburants. Cette dernière transformation nécessite le plus souvent l'utilisation d'enzymes sur des substrats complexes à l'état solide et semi-cristallins, comme l'amidon.

L'hydrolyse enzymatique de l'amidon intervient dans de nombreux processus naturels comme le métabolisme de l'amidon dans les plantes, la digestion ou certaines fermentations, et industriels comme la production de sirops de glucose ou de bioéthanol.

Le but de cette étude est de comprendre le mode d'action sur l'amidon natif cristallin d'une première amylase optimisée pour la production de bioéthanol, appelée ici RA, puis d'une deuxième enzyme plus optimisée pour la production de sirops de glucose, nommée ici AFA.
L'imagerie synchrotron proposée par la ligne de lumière DISCO permet de visualiser la localisation et la diffusion de RA et AFA dans des grains d'amidon de maïs au cours de leur hydrolyse, à partir de la fluorescence du tryptophane ${ }^{\star}$ contenu dans ces enzymes [3]. Cette fluorescence ne varie pas au cours de la réaction, car elle est liée à l'enzyme et non pas au substrat.

L'imagerie bidimensionnelle n'est pas suffisante pour suivre la localisation de l'enzyme dans le grain, ne permettant pas de distinguer si l'enzyme est en surface ou à l'intérieur du grain.

En reconstruisant la localisation tridimensionnelle de l'enzyme au cours du temps (fig. 4), il devient possible de mieux comprendre son mode de fonctionnement et plus particulièrement la façon dont la structure des grains d'amidon natifs peut être hydrolysée selon la source d'amylase utilisée. On remarque ainsi sur cette figure que l'enzyme entoure le grain. D'autres amylases commençaient par s'infiltrer dans les défauts du grain, avant de le digérer depuis l'intérieur.

\section{Second exemple : suivi de l'entrée de nanoparticules dans des cellules cancéreuses}

L'exposition orale aux nanoparticules est un problème de santé publique. L'utilisation croissante du dioxyde de titane $\left(\mathrm{TiO}_{2}\right)$ comme pigment alimentaire, mais aussi comme antibactérien, soulève des questions sur sa fixation à l'intérieur de l'organisme et des cellules. Jusqu'à présent, la localisation des nanoparticules de $\mathrm{TiO}_{2}$ s'effectuait soit avec un marquage secondaire, soit par microscopie électronique. L'utilisation d'une excitation ultraviolette lointaine permet d'observer directement la luminescence du $\mathrm{TiO}_{2}$, sans ajouter de marqueur de fluorescence secondaire, et sur des cellules vivantes, contrairement à la microscopie électronique.

Nous avons suivi l'entrée de ces nanoparticules dans des cellules immortalisées ${ }^{\star}$ (fig. 5a). Afin d'améliorer la détection subcellulaire, une tomographie a été réalisée et des plans sériés espacés de $100 \mathrm{~nm}$ ont été segmentés (fig. 5b). La tomographie permet de lever le doute sur la localisation des amas de nanoparticules. Les images bidimensionnelles ne permettent pas de discriminer entre une localisation intracellulaire et une localisation à la surface des cellules. La tomographie permet de confirmer la localisation intracellulaire.

\section{Conclusion}

La tomographie de fluorescence DUV permet de localiser en temps réel des objets ou des amas d'objets de petite taille (entre 50 et $500 \mathrm{~nm}$ ). Ces objets doivent présenter la propriété de réémettre de la lumière (fluorescence ou luminescence) après une excitation DUV. Cette méthode est très utile pour suivre les petites particules métalliques luminescentes, mais aussi des antibiotiques fluorescents telles les fluoroquinolones.

\section{Références \\ 1• N. Stuurman, N. Amodaj et R.D. Vale, Microscopy Today, 15 (2007) 42-43. \\ 2• F. Jamme et al., Microscopy and Microanalysis, 16 (2010) 507-514. \\ 3• G. Tawil et al., Anal. Chem., 83 (2011) 989-993.}

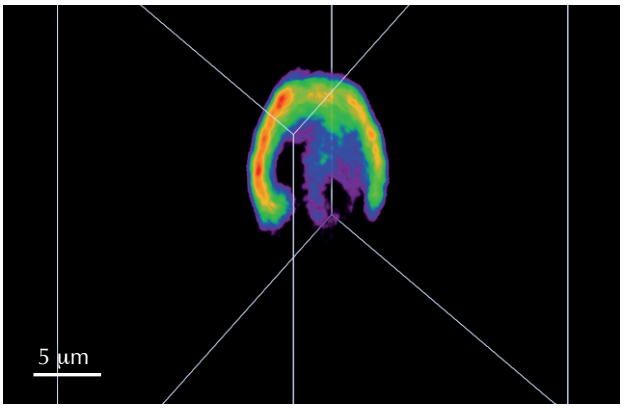

4. Reconstruction 3D de la localisation d'une enzyme (amylase) autour d'un grain d'amidon. La résolution spatiale, de l'ordre de $120 \mathrm{~nm}$, est compatible avec la taille du grain de l'ordre d'une dizaine de microns. Les couleurs chaudes correspondent aux maxima d'intensité lumineuse, que l'on peut relier à la localisation de l'enzyme autour du grain.
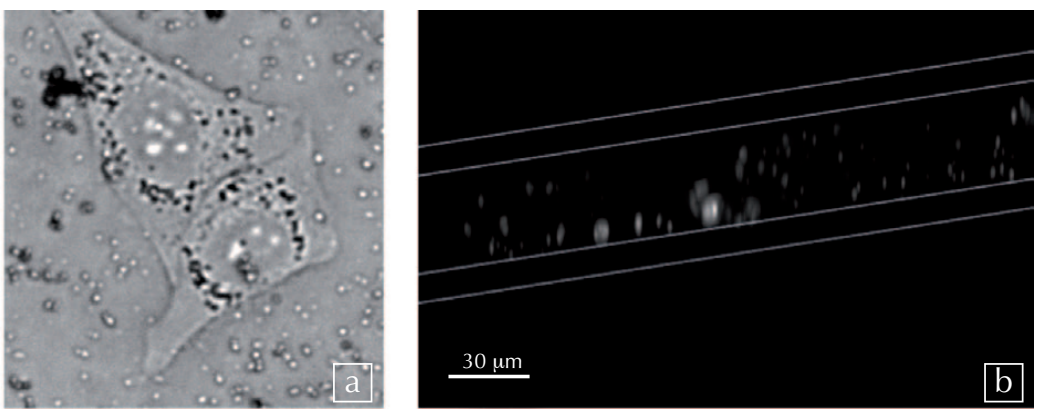

5. Segmentation d'une image de fluorescence de nanoparticules de $\mathrm{TiO}_{2}$ dans une cellule. (a) Les cellules, d'une taille de l'ordre d'une vingtaine de microns, ont absorbé des amas de nanoparticules d'environ $90 \mathrm{~nm}$ en couronnes.

(b) La tomographie permet de confirmer leur incorporation. (Les lignes blanches sont des lignes de fuite pour indiquer le volume et la perspective). 